\title{
Production and Characterization of Melon Sulfated Pectin (Cucumis melo var. acidulus) Nanoparticles with Anticoagulant Potential
}

\author{
Roberta Carolina Rangel de Oliveira ${ }^{1 *}$, Raimundo Rafael Almeida ${ }^{2}$, Antônia Flávia Justino Uchoa ${ }^{2}$, Ana Paula dos Santos ${ }^{1}$, \\ Nágila Maria Pontes Silva Ricardo ${ }^{2}$, Romélia Pinheiro Gonçalves Lemes' and Tamara Gonçalves de Araújo' \\ 'Department of Pharmacy, Federal University of Ceara, Fortaleza, Ceara, BRAZIL. \\ ${ }^{2}$ Department of Organic and Inorganic Chemistry, Polymers and Materials Innovation Laboratory (LabPIM), Federal University of Ceara, Campus do Pici, Fortaleza \\ CE, BRAZIL.
}

\begin{abstract}
Objective: Unfractionated heparin and low molecular weight heparin are the drugs most used today in coagulopathy. Although effective, these drugs have limitations in their usage, which aroused the interest of the scientific community in researching other substances with a similar effect. This study aims to analyze the anticoagulant activity of isolated and chemically modified pectin extracted from Caipira melon (Cucumis melo var. acidulus) and its nanoencapsulation for therapeutic use. Materials and Methods: For the analysis of anticoagulant activity, thrombin time, prothrombin time and activated partial thromboplastin time were performed. The nanoparticles were produced by a double emulsion using poly(lacticco-glycolic acid) and poly (vinyl alcohol) and, subsequently, were analyzed morphologically by scanning electron microscopy and characterized in the Zetasizer Nano ZS90. Results: The pectin tests showed promising anticoagulant activity. In order to increase the biological activity of this substance, nanoparticles of sulfated pectin were obtained, attaining particle size $301.03 \mathrm{~nm}$, polydispersity index of 0.18 , zeta potential of
\end{abstract}

$-16.63 \mathrm{mV}$ and Acid $\mathrm{pH}$, the results were considered satisfactory by the researchers. The encapsulation efficiency of the sulfated pectin was $76.31 \%$ with controlled release. The scanning electron microscopy (SEM) showed uniform and spherical nanoparticles. Conclusion: The results indicated that the sulfated pectin nanoparticles can be seen as an innovative alternative in anticoagulant therapy.

Keywords: Anticoagulant, Heparin, Nanoparticles, Sulfated polysaccharides. Correspondence

Roberta Carolina Rangel de Oliveira,

Department of Pharmacy, Federal University of Ceara, Fortaleza, Ceara, Brazil, Capitão Francisco Pedro, 1210-Rodolfo Teófilo, Fortaleza, Ceará, 60.430-170, BRAZIL.

Phone: +5585988233572

Email: robcarolina@hotmail.com

DOI: 10.5530/jyp.2020.12.27

\section{INTRODUCTION}

Unfractionated heparin (UFH) and its derived Low Molecular Weight Heparin (LMWH) are the chosen drugs for the treatment of thromboembolic disturbances. However, reports of adverse effects related to these drugs are frequent, such as hemorrhage, thrombocytopenia and osteoporosis, as well as problems such as structural diversity and contamination by animal pathogens, which justifies the growth by the search for new compounds with similar anticoagulant activities, fewer adverse effects and different of those which come from animal sources. ${ }^{1,2}$ Along the compounds that can be used as anticoagulant agents, the sulfated polysaccharides stand out, because the literature states that its property is related to the presence of sulfate groups and their distribution along the chain., ${ }^{2,3}$ Factors such as molecular weight, chemical composition, sulfate content and position and uronic acid content may interfere with the polysaccharide anticoagulant activity. Algae and invertebrates that have naturally sulfated polysaccharides in their constitution are already confirmed to possess anticoagulant and antithrombotic activity. ${ }^{1}$ However, as these species are limited, chemically polysaccharides sulfates may represent a good alternative. ${ }^{5}$

Aiming at the fruit valorization, the increase of the commercialization profits and the sustainable reuse, this work analyzed the anticoagulant action of the pectin in Caipira melon (Cucumis melo var. acidulus), which is widely produced in the Northeast of Brazil and possess irrelevant commercial value. It is a high molecular weight natural polysaccharide, biocompatible, non-toxic, anionic, already isolated and chemically sulfated in a previous study. ${ }^{1,6}$ Therefore, it was found that the viability of this sulfated pectin represents a new pharmaceutical alternative in the treatment of coagulation disorders.

Studies assert that the drug can be nanoencapsulated to improve its pharmacokinetic profile, improve treatment efficacy, minimize side effects and increase its chemical and conformational stability, confirming the efficiency of the nanoencapsulation of anticoagulant drugs. ${ }^{7,8}$ Therefore, the purpose of this paper was to evaluate the anticoagulant activity of pectin isolated and later chemically sulfated and, from these results, produce and characterize a nanocarrier system to expand its therapeutic potential.

\section{MATERIALS AND METHODS}

\section{Materials}

Samples of sulfated and non-sulfated polysaccharides were provided by the Department of Chemistry, at the Federal University of Ceara. Unfractionated heparin (Hemofol 5000UI / ml - Lote18030036) was purchased commercially. The Kits and the commercial kits used in the coagulation tests (APTT, PT and TT) were purchased commercially (Bios Diagnóstica ). Poly (lactic-co-glycolic acid) (PLGA) with a 50:50 lactide co-glycolide ratio (MW 40-75 kDa) and poly (vinyl alcohol) (PVA) (MW 13-23 kDa, 87-89\% hydrolyzed) were purchased from Sigma-Aldrich. 
Samples Blood: the samples for plasma separation used in in vitro tests were obtained from donors from the Hemocenter of Ceara (HEMOCE), obeying the following criteria: adult donors who do not take any type of medicine; healthy and donors of both sexes. Samples from hemolyzed patients were excluded. The project was approved by the Research Ethics Committee of the Federal University of Ceara, with the protocol number 1.572.008.

\section{Assay of anticoagulant activity}

The Activated Partial Thromboplastin Time (APTT) clotting assay was carried out according to the method of Castro et al., ${ }^{9}$ using normal human plasma. In the assay, plasma samples $(90 \mu \mathrm{L})$ were mixed with different amounts of polysaccharide in $0.9 \% \mathrm{NaCl}(10 \mu \mathrm{L})$ and incubated at $37^{\circ} \mathrm{C}$ for 60 seconds and prewarmed at $100 \mu \mathrm{L}$. APTT assay reagent was added and allowed to incubate at $37^{\circ} \mathrm{C}$ for 2 minutes. Thenceforth, the substance was prewarmed and $0.25 \mathrm{M}$ calcium chloride $(100 \mu \mathrm{L})$ was then added. The APTT recorded as the time for clot formation in a coagulometer. For thrombin time (TT) clotting assay, citrated normal human plasma $(90 \mu \mathrm{L})$ was mixed with $10 \mu \mathrm{L}$ of a solution of polysaccharide and incubated at $37^{\circ} \mathrm{C}$ for 60 seconds. Then, $200 \mu \mathrm{L}$ of TT assay reagent preheated to $37^{\circ} \mathrm{C}$ was added and the clotting time was recorded one more time. Prothrombin time (PT) clotting assay was performed as follows: citrated normal human plasma $(90 \mu \mathrm{L})$ was mixed with $10 \mu \mathrm{L}$ of a solution of polysaccharide and incubated at $37^{\circ} \mathrm{C}$ for $1 \mathrm{~min}$. Then, $200 \mu \mathrm{L}$ of $\mathrm{PT}$ assay reagent pre-incubated at $37^{\circ} \mathrm{C}$ for 10 mins was added and clotting time was recorded. The bioassay results were expressed as means \pm standard deviation (SD). The experimental data were subjected to an analysis of variance for a completely random design and three samples were prepared for assays of every attribute.

\section{Preparation of nanoparticles}

The NSP was prepared according to the method described by Chereddy et al..$^{10}$ considering some modifications. Briefly, $100 \mu$ l sulfated pectin $(1 \mathrm{mg} / \mathrm{ml}$ ) was then discarded in the $100 \mathrm{mg} 50: 50$ solution of PLGA (poly (lactic-co-glycolic acid)), in $5 \mathrm{ml}$ dichloromethane (DCM) and sonicated for 15 seconds (50\% Amplitude, 5 seconds with pulse on and 2 seconds with pulse off), using an ultrasonic processor (Sonifier model W-450D Branson). Once prepared, this first emulsion was then poured into $100 \mathrm{mg}$ PVA, dissolved in $10 \mathrm{ml}$ deionized water and sonicated again for 15 seconds (Amplitude 50\%, pulse 5 seconds "on" and 2 seconds "off”) using the processor again (Sonifier ${ }^{\circ}$ Model W $-450 \mathrm{D}$ Branson). The formed water/oil/water double emulsion $(\mathrm{w} / \mathrm{o} / \mathrm{w})$ was then stirred using a magnetic stirrer, to remove excess DCM for 6 hours. The nanoparticles were separated by centrifugation (5810R Centrifuge, Eppendorf, USA) at 3.000rpm for 15 minutes, using a separation filter ( $15 \mathrm{ml}$ Amicon Ultra $100 \mathrm{KDa}$ ) and finally, the sample was lyophilized (Labconco, USA). Empty nanoparticles were prepared with the same procedure, considering the addition of sulfated pectin during formulation preparation. ${ }^{11,12}$

\section{Characterization of Nanoparticles}

The mean particle size (nm), the polydispersity index (PDI) and the Zeta potential (ZP) of NSP were determined using a Zetasizer Nano ZS90 instrument (Malvern Instruments, Malvern, UK) at $25^{\circ} \mathrm{C}$, by dynamic light scattering (DLS). All measurements were carried out in triplicate $(n=3)$.

\section{Scanning Electron Microscopy (SEM)}

The particles were characterized by Scanning Electron Microscopy (SEM) (Quanta 450 FEG, FEI Company, USA) at a voltage of $30 \mathrm{kV}$, after prior coating with the silver under vacuum by sputtering using a QT150 ES (Quorum Technologies, USA) apparatus.

\section{Differential Scanning Calorimetry (DSC)}

The physical state of sulfated pectin inside the nanoparticles was characterized by the analysis of the DSC curves. The curves were obtained in DSC cell (Shimadzu, Kyoto, Japan) using aluminum crucibles with approximately $5 \mathrm{mg}$ of samples, under dynamic air atmosphere $(40 \mathrm{~mL} / \mathrm{min})$ and a heating rate of $10^{\circ} \mathrm{C} / \mathrm{min}$ at a temperature range from $25^{\circ} \mathrm{C}$ to $600^{\circ} \mathrm{C} .13$

\section{Thermogravimetric Analysis (TG) and derived thermogravimetry (DTG)}

The thermal stability of the samples was verified by simultaneous TG and DTG, using a Q 50 TGA (TA INSTRUMENTS). TG-DTG curves were obtained in the temperature range from $30^{\circ} \mathrm{C}$ to $800^{\circ} \mathrm{C}$, using aluminum crucibles with approximately $2 \mathrm{mg}$ of samples, under dynamic air atmosphere $(40 \mathrm{~mL} / \mathrm{min})$ and heating rate of $10^{\circ} \mathrm{C} / \mathrm{min}^{.14}$

\section{Determination of Entrapment Efficiency (EE)}

The calculation of the quantity of nanoencapsulated substance was indirect; first the SP present in the supernatant was quantified using a method based on the linearity of the APTT results of increasing concentrations of this pectin in the plasma of healthy patients. The dosedependent linearity of APTT in the analyzed concentrations made it viable to establish a dose-response curve that, then, made it possible to quantify the free polysaccharide. Later, the formula below permitted the calculation of these nanoencapsulated substances. ${ }^{14,15}$

$$
\mathrm{EE}(\%)=\frac{\text { Total initial drug }- \text { Drug in supernatant }}{\text { Total initial drug }} \times 100
$$

\section{Stabilities studies}

The samples were stored in room temperature and sheltered from light and heat for 30 days. To evaluate the stability of the obtained nanoparticles, the following parameters were monitored: Zeta potential $(\mathrm{mV})$, polydispersity index, particle size $(\mathrm{nm})$ on a Zetasizer Software Malvern Version 7.12, by dynamic mirroring of light at times 0 and 30 days. The $\mathrm{pH}$ of the samples was also analyzed in both times using $\mathrm{pH}$ meter Quimis, Q400AS.

\section{Nanoparticles release study}

Initially, 30mg NSP of lyophilized sulfated pectin was dispersed in $3 \mathrm{ml}$ of phosphate-buffered saline (PBS). The NP were incubated at room temperature while stirring for 6 days. After 1 and 2 hours and after 1, 2 and 5 days, $1 \mathrm{ml}$ of the supernatant was collected from the environment and filtered on an ultracentrifugation tube and the same PBS was reposted on the releasing environment. The amount of sulfated pectin released on determined times was calculated through the dose-response curve already described on the Encapsulation Efficiency tests. ${ }^{10}$

\section{Rheology study of SP and UFH (Unfractionated Heparin) solutions}

The study of the rheological behavior of UFH solutions using the AR 2000 controlled tension rheometer (TA INSTRUMENTS), with a conical plate geometry that possesses a diameter of $40 \mathrm{~cm}$ and angle 10 ' 47 ', maintaining a range of $27 \mu \mathrm{m}$. The solutions of SP, nanoparticles of SP and UFH $(0.25 \mathrm{mg} / \mathrm{ml})$ were placed in the container and the rate of shear stress applied by gradual rotation of the rod measured the torque required to calculate the shear stress from 1 to $1000 \mathrm{~s}^{-1}$. The samples were shaken for approximately 2 minutes before the beginning of the experiment. A comparison was made between the rheological properties of the analyzed samples and all experiments were done in triplicate. 


\section{Statistical Analysis}

All obtained results were analyzed by the Shapiro-Wilk test to measure the normality distribution of data in which showed parametric and non-parametric distributions. Therefore, the results were presented as the meanor median \pm measures of dispersion. The statistical differences between the groups were calculated using the $t$ test, Analysis of Variance (ANOVA) test followed by the Bonferroni posttest or their correspondent non-parametric tests, considering a critical alpha of 0.05 significance level. The program used for was GraphPad Prism, version 5.01.

\section{RESULTS}

\section{In vitro coagulation tests}

According to Figure 1, sulfation has interfered with the anticoagulant activity and the isolated pectin shows no anticoagulant Oliveiraeffect. The APTT result of the SP was statistically similar to UFH, used as a positive control. $(p<0.001)$.

\section{Anticoagulant activity of sulfated pectin}

Observing the results of Table 1 . It was verified the alterations on the TT, as expected, since the APTT results were extended dose-dependently. The prothrombin time (PT) shows no significant change.

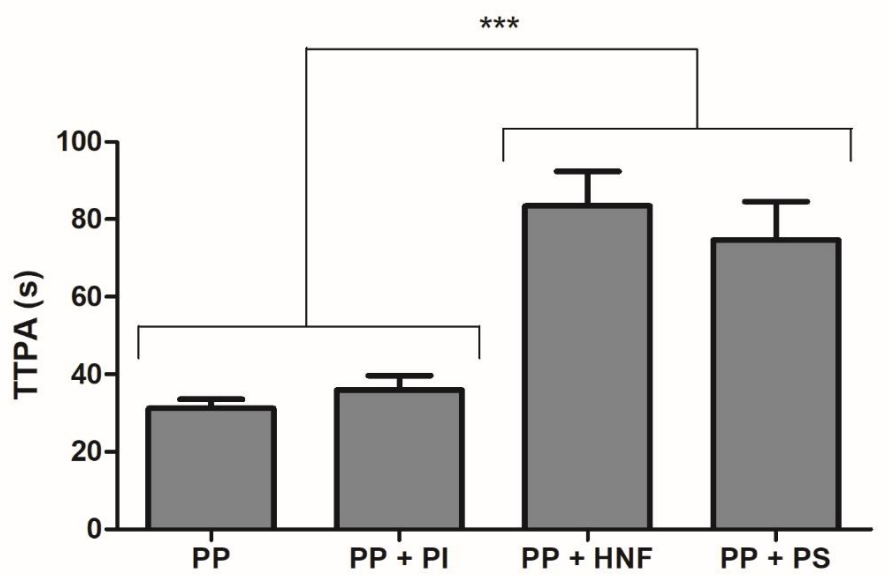

Figure 1: The activated partial thromboplastin time (APTT) in seconds of pure plasma (PP) in vitro solution combined with isolated pectin $0.5 \mathrm{ml} / \mathrm{mL}(\mathrm{P}+\mathrm{IP})$, unfractionated heparin $(P+U F H)$ and sulfated pectin $0.5 \mathrm{mg} / \mathrm{mL}(P+S P)$. The data are presented as mean \pm SD of six independent replicates and statistically compared using the ANOVA followed by Bonferroni posttest. Significant differences: $p<0.001^{* * *}$.
The E50 of the SP was calculated from the statistical analysis of the data in Table 1. The confidence interval of the active dose of SP able to achieve $50 \%$ of the maximum anticoagulant effect is between 0.25 and $0.40 \mathrm{mg} / \mathrm{ml}$.

\section{Development and characterization of the nanoparticles}

The double emulsion method with the evaporation of the solvent used in this study allows to achieve homogeneous nanoparticles. All samples have shown average diameters of particles (particle size) of $301.00 \pm 7.19 \mathrm{~nm}$ and the polydispersity index of the samples was $<0.2$.

Both nanoparticles of SP have shown negative superficial electrical density (zeta potential).

\section{Morphology analysis}

In Figure 2 it is possible to observe spherical, regular nanoparticles in the range of $300 \mathrm{~nm}$ average diameter.

\section{Entrapment efficiency (EE)}

In this study, the EE achieved for the NPS was $76.4 \%$.

\section{Release studies}

In Figure 3, it is possible to observe the maximum sulfated pectin (SP) release, reaching approximately $49 \%$ in 5 days.

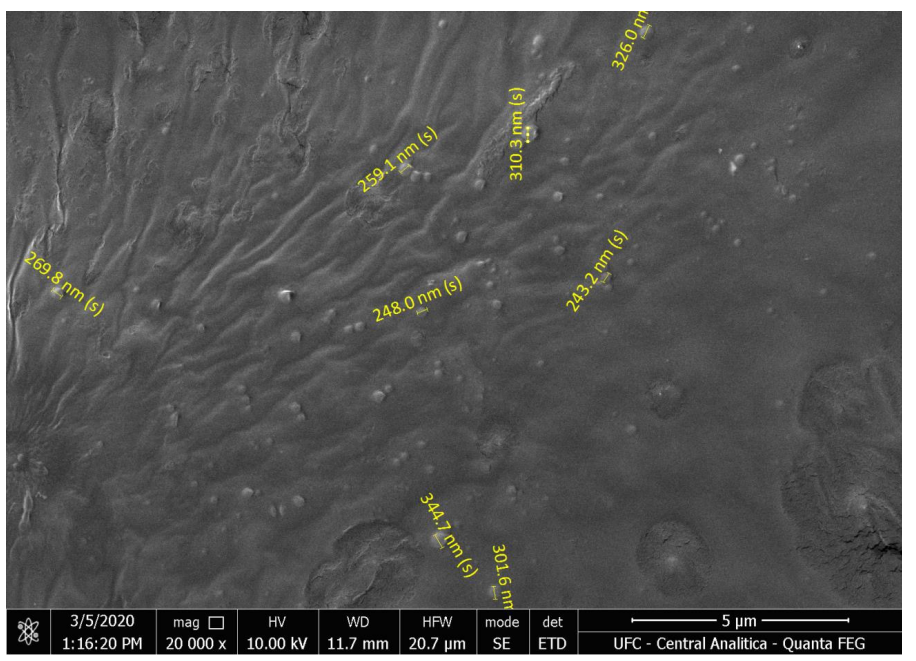

Figure 2: Scanning electron microscope images of sulfated pectin nanoparticles showed nano sized spherical particles.

Table 1: Prothrombin time (PT), activated partial thromboplastin time (APTT) and thrombin time (TT) of pure plasma solutions alone or in association with isolated pectin $(\mathrm{PP}+\mathrm{PI})$ and pure plasma with sulfated pectin $(\mathrm{PP}+\mathrm{PS})$ in six concentrations studied.

\begin{tabular}{|c|c|c|c|c|c|c|}
\hline$(\mathrm{mg} / \mathrm{ml})$ & APTT & PT & & & & \\
\hline & seconds & p-value & seconds & $\mathrm{p}$-value & seconds & p-value \\
\hline Plasma puro (PP) & $31.21 \pm 0.7$ & & $14.36 \pm 0.4$ & & $18.2 \pm 0.7$ & \\
\hline PP + PI 1,0 & $35.2 \pm 0.5$ & $p<0.01$ & $14.34 \pm 0.9$ & NS & $20.1 \pm 0.6$ & NS \\
\hline$P P+P S 1,0$ & $114.76 \pm 1.3$ & $p<0.001$ & $14.06 \pm 0.8$ & NS & $87.2 \pm 0.9$ & $\mathrm{p}<0.001$ \\
\hline $\mathrm{PP}+\mathrm{PS} 0,75$ & $92.13 \pm 1.7$ & $p<0.001$ & $14.03 \pm 0.9$ & NS & $66.7 \pm 3.3$ & $\mathrm{p}<0.001$ \\
\hline$P P+P S 0,5$ & $74.43 \pm 2.1$ & $p<0.001$ & $14.56 \pm 0.6$ & NS & $56.6 \pm 2.0$ & $\mathrm{p}<0.001$ \\
\hline $\mathrm{PP}+\mathrm{PS} 0,25$ & $58.4 \pm 1.6$ & $p<0.001$ & $14.03 \pm 1.0$ & NS & $24.3 \pm 1.8$ & $\mathrm{p}<0.001$ \\
\hline $\mathrm{PP}+\mathrm{PS} 0,125$ & $46.06 \pm 0.9$ & $p<0.001$ & $13.76 \pm 1.5$ & NS & $19.9 \pm 2.7$ & NS \\
\hline$P P+P S 0,0625$ & $37.3 \pm 1.0$ & NS & $13.65 \pm 0.4$ & NS & $19.1 \pm 1.6$ & NS \\
\hline
\end{tabular}

Values are represented as means $\pm \mathrm{SD}(\mathrm{n}=3)$. Significant differences compared to plasma pure alone using ANOVA followed by Bonferroni posttest. NS: not significant. 


\section{Thermogravimetric analysis of NPS}

Figure 4 shows the presence of three endothermic peaks: a first event in the temperature range of $69.11^{\circ} \mathrm{C}$ followed by two consecutive events at $193.7^{\circ} \mathrm{C}$ and $278.1^{\circ} \mathrm{C}$. No crystallinity event was detected in relation to the encapsulated drug. The TG-DTG curve has confirmed the data achieved on DSC. The nanoparticles show thermal stability on the temperature range of $190^{\circ} \mathrm{C}$ when compared to the pure polymer. Weight loss occurs in two stages: $314^{\circ} \mathrm{C}-58.38 \%$ and $432-32.38 \%$, associated with exothermic events between the temperatures of $206^{\circ} \mathrm{C}$ and $499^{\circ} \mathrm{C}$.

\section{Stability tests}

According to the analysis made, the nanoparticles of sulfated pectin (NSP) remained stable for 30 days at room temperature and sheltered from light and heat, as it is shown in Table 2. The formulations developed

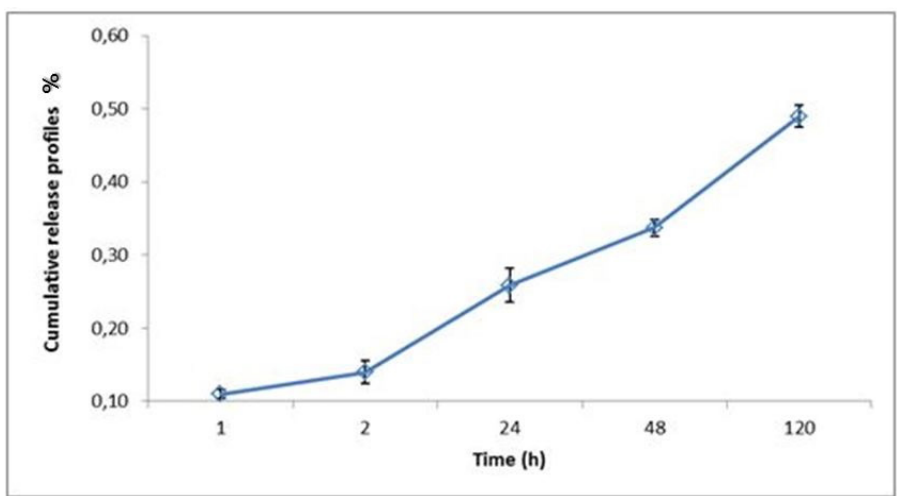

Figure 3: Cumulative release profiles of sulfated pectin nanoparticles in phosphate-buffered saline $(\mathrm{pH}=7.4)$ at $37^{\circ} \mathrm{C}$.

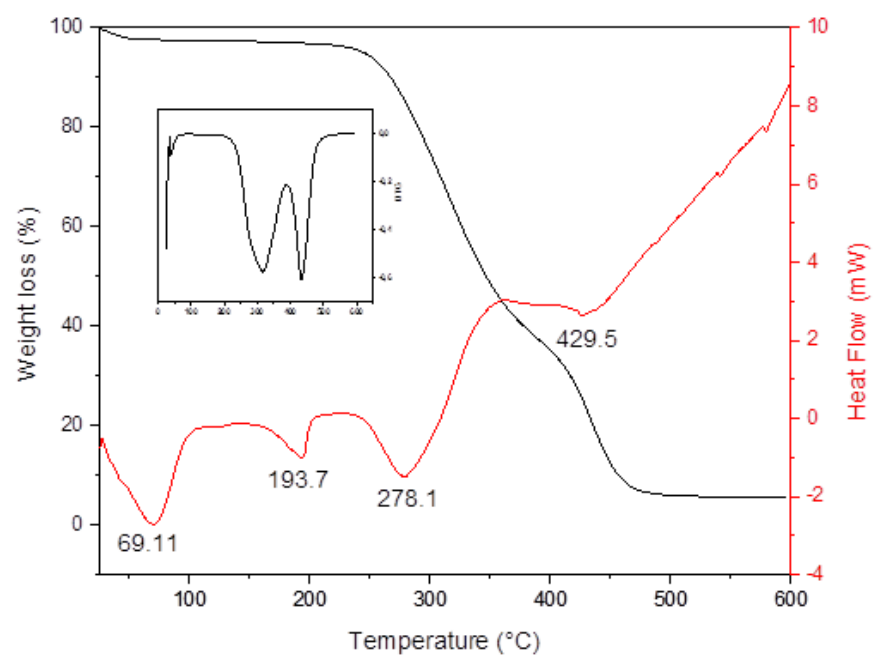

Figure 4: Thermogravimetric analysis (TGA) and differential scanning calorimetry (DSC) of NSP. (The derivative graph is embedded). show no significant difference compared to the parameters analyzed in the evaluated times.

\section{Rheological behavior studies}

It was possible to observe the similarity of rheological behavior of sulfated pectin (SP), nanoparticles of sulfated pectin (NSP) and unfractionated heparin (UFH) aqueous solutions in a concentration of $0.25 \mathrm{mg} / \mathrm{ml}$.

\section{DISCUSSION}

According to the results, it was possible to verify that the chemical sulfation of the sulfated pectin promoted its anticoagulant activity. Studies ${ }^{1,2,16}$ assert that the introduction of sulfate groups has a direct influence on antithrombotic and anticoagulant activities. Similar results were obtained by $\mathrm{Hu}^{5}$ et al. and Chaouch et al. ${ }^{16}$ that chemically sulfated other pectins and promoted an extension in the APTT. Along with these studies, the non-sulfated pectin showed no anticoagulant activity as well. For these results, APTT, TT and PT in vitro stitching tests were performed.

It is known that the APTT assesses intrinsic pathway coagulation factors. TP is used to characterize extrinsic coagulation factors, while an increase in TT suggests inhibition of thrombin or fibrin production. ${ }^{17,18}$ Looking at the results in Table 1. It was found the changes in TT and APTT, with APTT altering in a dose-dependent manner, as described by Cui et al. ${ }^{19}$ and Wang et al. ${ }^{20}$ in similar works.

As the TP displayed no significant change, it was concluded that the samples involved in these tests acted only in the intrinsic coagulation pathway. This result corroborates with the research by Adrien et al. ${ }^{4}$ and Da Silva et al., ${ }^{21}$ where sulfated polysaccharides were also subjected to these tests and only significant variations in the APTT were verified. These authors state that the intrinsic pathway is the main target of sulfated polysaccharides and the extrinsic pathway is not affected by these substances. ${ }^{9}$

For the nanoparticles obtained, the particle size is one of the most important parameters, as it determines the biocompatibilities and bioactivities of the nanoparticles, in addition to possessing direct relevance to the stability of the formulation..$^{22}$ Similar results have been reported in other studies that reached $195 \mathrm{~nm}$ to $251 \mathrm{~nm}$ nanoparticles. ${ }^{10}$ In a similar test, medium-sized nanoparticles $512.8 \mathrm{~nm}$ and $269 \mathrm{~nm}$ were produced for nanoencapsulated low molecular weight heparin, using PVA and PLGA as well. ${ }^{12,23}$

Regarding low PDI, the literature confirms that low values reveal good homogeneity of the samples. ${ }^{21}$ Already analyzing the zeta potential, this parameter is probably related to the free carboxylic groups on the surface. ${ }^{24}$ According to Sun et al..$^{22}$ in the case of charged particles, the greater the Zeta potential, the greater the repulsive interaction, leading to the formation of more stable particles with a more uniform size distribution.

According to MEV and EE, the results were similar to others reported in the literature regarding the production of sulfated polysaccharide nanoparticles using PVA and PLGA..$^{25,26}$

According to the authors, the efficiency of encapsulation is related to the concentration of polymers and surfactants. As the concentration of

Table 2: Stability of the nanoparticles of sulfated pectin (NSP) in terms of zeta potential (mV), polydispersity index, particle size (nm) and pH, for 30 days.

\begin{tabular}{cccccc}
\hline Day & Zeta potential $(\mathrm{mV})$ & Polydispersity index & Particle size $(\mathrm{nm})$ & $\mathrm{pH}$ & $\mathrm{p}$-value \\
\hline 1 & $-16.63 \pm 1.96$ & $0.18 \pm 0.04$ & $301 \pm 7.19$ & $4.20 \pm 0.01$ & $\mathrm{p}>0.05$ \\
30 & $-14.22 \pm 0.51$ & $0.14 \pm 0.02$ & $304 \pm 3.59$ & $4.40 \pm 0.08$ & \\
\hline
\end{tabular}

Values are represented as means $\pm S D(n=3)$. Significant differences between day 1 and day 30 using ANOVA followed by Bonferroni posttest. Not significant: $\mathrm{p}>0.05$. 
surfactants increases, the size of the particles and the surface area decrease, which leads to an increase in the release of drugs by the nanoparticles. ${ }^{11}$ Analyzing this release, it can be found that in the first two hours it was faster. However, over time, the speed decreased. Therefore, with a longer release, the drug remained in the body for a longer time, because one of the main disadvantages of heparins is its low bioavailability and the need for many daily doses. ${ }^{8}$

The DSC curves of the NPS show that the first event may be related to the release of acetyl groups that have been transformed into acetic acid and the successive catalytic degradation of the main chain at higher temperatures. The TG-DTG curve revealed no events related to the melting point of the drug. This fact reinforces the idea that it is encapsulated in amorphous nanoparticles, as confirmed by DSC. ${ }^{1,25}$

As for stability, it is relevant to emphasize that monitoring $\mathrm{pH}$ over time is important. Decreasing $\mathrm{pH}$ values may indicate the beginning of polymer degradation. ${ }^{27}$ According to the results obtained, all formulations pointed acid $\mathrm{pH}(4.0-4.5)$ for 30 days due to the negative characteristics of the sulfated polysaccharides and, therefore, remained stable. ${ }^{12}$

The results of the rheological behavior clarified as well that the NSP in the studied concentration has a Newtonian character, since the relationship between shear stress and local velocity gradient is linear, with the proportionality being constant to the viscosity of the fluid. Similar behavior is demonstrated by UFH and SP, suggesting that the solution with SP nanoparticles can be administered intravenously.

\section{CONCLUSION}

In vitro and in vivo studies of the applications of sulfated polysaccharides as anticoagulants are increasing and expanding among the scientific community. They are receiving significant attention from the pharmaceutical market. Due to the problems shown by the use of heparins, the chemically modified SP extracted from Japanese melon can be a new alternative to non-toxic, efficient and accessible anticoagulant therapy.

Coagulation tests results were promising. The nanoencapsulation methodology was successful with satisfactory encapsulation efficiency. The nanoparticles produced are stable, homogeneous, possess controlled release and rheological behavior similar to UFH.

The small number of articles found about the species of sulfated pectin used as anticoagulants shown the need for a continuous effort of researchers and pharmaceutical industry to increase the studies of these species and, eventually, lead them to the development of an innovative, safe and efficient drug.

\section{ACKNOWLEDGMENTS}

The Coordination of Improvement of Higher Education Personnel (CAPES)/Ministry of Education (MEC). The authors would like to thank the Central Analítica-UFC/CT-INFRA/MCTI-SISANO/CAPES for the support.

\section{CONFLICT OF INTEREST}

The authors declare no conflicts of interest.

\section{ABBREVIATIONS}

APTT: Activated Partial Thromboplastin Time; DCM: Dichloromethane; DLS: Dynamic Light Scattering; DSC: Differential Scanning Calorimetry; EE: Entrapment efficiency; HEMOCE: Hemocenter of Ceará; IP: Isolated pectin; LMWH: Low Molecular Weight Heparin; MW: Molecular weight; NSP: Nanoparticles of sulfated pectin; PBS: Phosphate-buffered saline; PDI: Polydispersity index; PLGA: Poly (lactic-co-glycolic acid); PP: Pure plasm; PT: Prothrombin time; PVA: poly (vinyl alcohol); SD: Standard deviation; SEM: Scanning Electron Microscopy; SP: Sulfated Pectin; TG: Thermogravimetric Analysis; TT: Thrombin time; UFH: Unfractionated Heparin; ZP: Zeta potential.

\section{REFERENCES}

1. Sousa AL, Ribeiro AC, Santos DG, Ricardo NM, Ribeiro ME, Cavalcanti ES, et al. Chemical modification of common melon pectin (Cucumis melo var. acidulus). Quim Nova. 2017;40(5):554-60.

2. Oliveira BHP, Gracher AHP, Tosin SFF, lacomini M, Cipriani TR. Anticoagulant activity of native and partially degraded glycoglucuronomannan after chemical sulfation. Int J Biol Macromol. 2015;80:328-33.

3. Oliveira RCR, Almeida RR, Gonçalves TA. A review of plant sulfated polysaccharides and their relations with anticoagulant activities. J Dev Drugs. 2016;5(3):3-5

4. Adrien A, Dufour D, Baudouin S, Maugard T, Bridiau N. Evaluation of the anticoagulant potential of polysaccharide-rich fractions extracted from macroalgae. Nat Prod Res. 2017;31(18):2126-36.

5. Hu $Y, Y e X$, Yin $X$, Chen S. Sulfation of citrus pectin by pyridine-sulfurtrioxide complex and its anticoagulant activity. LWT- Food Sci Technol. 2015;60(2):1162-7.

6. Chen J, Liu W, Liu CM, LiT, Liang RH, Luo S. Pectin modifications: a review. Crit Rev Food Sci Nutr. 2015;55(12):1684-98

7. Ibrahim SS, Osman R, Awad GA, Mortada ND, Geneidy AS. Low molecular weight heparins for current and future uses: approaches for micro-and nanoparticulate delivery. Drug deliv. 2016;23(8):2661-7.

8. Akhtar F, Wan X, Wu G, Kesse S, Wang S, He S. Low-Molecular-Weight Heparins: Reduced Size Particulate Systems for Improved Therapeutic Outcomes. Molecules. 2018;23(7):1757.

9. Castro LSEPW, Sousa PT, Castro AJG, Santos MDSN, Soriano EM, Leite EL. Potential anti-angiogenic, antiproliferative, antioxidant and anticoagulant activity of anionic polysaccharides, fucans, extracted from brown algae Lobophora variegata. J. Appl. Phycol. 2015;27(3):1315-25.

10. Chereddy KK, Her CH, Comune M, Moia C, Lopes A, Porporato PE, et al. PLGA nanoparticles loaded with host defense peptide LL37 promote wound healing J Control Rel. 2014;194:138-47.

11. Jogala S, Rachamalla SS, Aukunuru J. Development of subcutaneous sustained release nanoparticles encapsulating low molecular weight heparin. J Adv Pharm Technol Res. 2015;6(2):58.

12. Pazzini C, Marcato PD, Prado LB, Alessio AM, Höehr NF, Montalvão S, et al. Polymeric nanoparticles of enoxaparin as a delivery system: in vivo evaluation in normal rats and in a venous thrombosis rat model. J Nanosci Nanotechnol. 2015;15(7):4837-43.

13. Mainardes RM, Gremião MPD, Evangelista RC. Thermoanalytical study of praziquantel-loaded PLGA nanoparticles. Rev Brasileira Ciências Farmacêuticas. 2006;42(4):523-530.

14. Marotta-Oliveira SSA, Marchetti JM. Determination of fractionated heparin in pharmaceutical dosage forms using turbidimetry. Quim Nova. 2011;34(6):1063-7.

15. Ibrahim SS, Osman R, Mortada ND, Geneidy AS, Awad GA. Passive targeting and lung tolerability of enoxaparin microspheres for a sustained antithrombotic activity in rats. Drug Deliv. 2017;24(1):243-51.

16. Chaouch MA, Hammi KM, Dhahri M, Mansour MB, Maaroufi MR, Cerf D, et al. Access to new anticoagulant by sulfation of pectin-like polysaccharides isolated from Opuntia ficus indica cladodes. Int J Biol Macromol. 2018:120:1794-800.

17. Liang L, Ao L, Ma T, Ni Y, Liao X, Hu X, et al. Sulfated modification and anticoagulant activity of pumpkin (Cucurbita pepo, Lady Godiva) polysaccharide. Int J Biol Macromol. 2018;106:447-55.

18. Dore CMPG, Alves MGDCF, Will LSEP, CostaTG, Sabry DA, Rêgo SLAR, et al. A sulfated polysaccharide, fucans, isolated from brown algae Sargassum vulgare with anticoagulant, antithrombotic, antioxidant and anti-inflammatory effects. Carbohydr Polym. 2013;91(1):467-75.

19. Cui J, Li Y, Wang S, Chi Y, Hwang H, Wang P. Directional preparation of anticoagulant-active sulfated polysaccharides from Enteromorpha prolifera using artificial neural networks. Scientific reports. 2018;8(1):3062.

20. Wang Y, Shao J, Yao S, Zhang S, Yan J, Wang H, et al. Study on the antithrombotic activity of Umbilicaria esculenta polysaccharide. Carbohydrate polymers. 2014; 105:231-6

21. Silva LC, Garcia T, Mori M, Sandri G, Bonferoni MC, Finotelli PV, et al. Preparation and characterization of polysaccharide-based nanoparticles with anticoagulant activity. Int J Nanomed. 2012;7:2975.

22. Sun SB, Liu P, Shao FM, Miao QL. Formulation and evaluation of PLGA nanoparticles loaded capecitabine for prostate cancer. I J Clin Exper Med. 2015;8(10):19670

23. Prado LB, Huber SC, Barnabé A, Bassora FDS, Paixão DS, Duran N, et al. Characterization of PCL and chitosan nanoparticles as carriers of enoxaparin and its antithrombotic effect in animal models of venous thrombosis. J Nanotechnol. 2017;(2):1-7.

24. Al-Nemrawi N, Alshraiedeh NA, Zayed A, Altaani B. Low molecular weight chitosan-coated PLGA nanoparticles for pulmonary delivery of tobramycin for 
cystic fibrosis. Pharmaceuticals. 2018;11(1):28

25. Fonseca LB, Nele M, Volpato NM, Seiceira RC, Pinto JC. Production of PMMA nanoparticles loaded with praziquantel through "in situ" miniemulsion polymerization. Macromol React Eng. 2013;7(1):54-63.

26. Peres LB, Peres LB, Araújo $\mathrm{PHH}$, Sayer C. Solid lipid nanoparticles for encapsulation of hydrophilic drugs by an organic solvent free double emulsion technique. Colloids and Surfaces B: Biointerfaces. 2016;140:317-23.

27. Ciocheta TM, Bender EA, Colomé LM. Desenvolvimento, caracterização e estabilidade de nanopartículas poliméricas contendo óleo de romã. Anais do Salão Internacional de Ensino, Pesquisa e Extensão. 2018;9(2):11.

Article History: Submission Date : 10-01-2020; Revised Date : 18-03-2020; Acceptance Date : 10-06-2020.

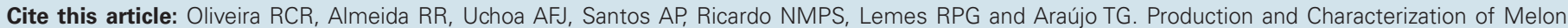
Sulfated Pectin (Cucumis melo var. acidulus) Nanoparticles with Anticoagulant Potential. J Young Pharm. 2020;12(2):135-40. 